Research Article

\title{
Quantum Dot Conjugated Magnetic Nanoparticles for Targeted Drug Delivery and Imaging
}

\author{
Indu Venugopal $^{1}$, Sebastian Pernal ${ }^{1}$, Taylor Fusinatto ${ }^{1,2}$, David Ashkenaz ${ }^{1,3}$, Andreas Linninger $^{1}$ \\ ${ }^{1}$ University of Illinois at Chicago, Department of Bioengineering, Laboratory for Product and Process Design \\ ${ }^{2}$ NSF-RET fellow from Bessie Rhodes Magnet School, Science Department \\ ${ }^{3}$ NSF-RET fellow from Innovations High School, Science Department \\ Corresponding author: E-mail: linninge@uic.edu
}

Received: Dec. 12, 2015; Accepted: Feb. 18, 2016; Published: Mar. 12, 2016.

Citation: Indu Venugopal, Sebastian Pernal, Taylor Fusinatto, David Ashkenaz and Andreas Linninger. Quantum Dot Conjugated Magnetic Nanoparticles for Targeted Drug Delivery and Imaging. Nano Biomed. Eng. 2016 8(I), 24-38.

DOI: $10.5101 /$ nbe.v8il.p24-38.

\begin{abstract}
Nanotechnology is being increasingly applied for developing drug delivery options for specific treatments. Magnetic nanoparticles have drawn attention as drug delivery vehicles due to their stability, biocompatibility and ability to be non-invasively guided to desired target areas using magnetic fields. In this paper, we describe a new delivery vehicle for magnetic drug targeting. In magnetic drug targeting, drug functionalized magnetic nanoparticles are guided and localized at specific sites using external magnetic fields. Magnetic nanoparticles act as contrast agents for magnetic resonance imaging. However, it cannot be visualized via this technique during drug delivery. This is between magnetic fields used for imaging and delivery can interference with each other. Our laboratory has synthesized a magnetic drug targeting vehicle conjugated with quantum dots that can be imaged during the drug delivery process with in vivo imaging techniques such as fluorescence molecular tomography. These nanocomposites can be used as drug delivery vehicles for the central nervous system, where drug targeting is especially difficult and minimizing side effects is critical.
\end{abstract}

Keywords: Fluorescent nanoparticles; Magnetic nanoparticles; Magnetic drug targeting; Drug delivery

\section{Introduction}

Along with the discovery and development of therapeutics molecules, one of the main challenges for disease treatment is the difficulty to deliver them to target sites within the body. Drug delivery to specific organs or tissues, especially to the Central Nervous System (CNS), poses significant challenges such as achieving high efficacy while evading side effects [1, 2]. Currently, the lack of specificity towards the target site generates the need for using high drug dosages to attain required therapeutic effect. However, this elevates the risk of toxicity in non-targeted regions, leading to unwanted side effects. For example, high doses of chemotherapeutic drugs are known to cause unacceptable systemic toxicity [3-6].

A significant advancement has been the development of nanoparticles (NPs) for delivering drugs. Variation in their biochemistry and material properties can create innovative therapeutic options for site-specific drug delivery, which takes advantage of interactions between target molecules with cellular and subcellular structures [7-12]. Targeted delivery reduces 
drug dosage and minimizes systemic toxicity, while maintaining therapeutic efficacy. The large surface area to volume ratio of nanoparticles, provides an ideal platform for drug loading and surface modifications for site specific targeting [13-18].

The small size of the NPs allows them to circumvent some of the most impenetrable barriers of the human body. For example, the blood brain barrier (BBB), which is formed by a continuous layer of endothelial cells joined together by tight junctions (zonulae occludens) surrounding blood vessels [19, 20], severely restricts access of large therapeutic molecules to the brain and spinal cord tissue. Previously, NP systems have been utilized to by-pass the BBB for treatment of CNS diseases like gliomas [21-23]. NP-based drug delivery systems also offer other benefits such as increased drug bioavailability [24-26], higher stability for treatments involving controlled release of drugs [27-29], effective delivery of poorly water soluble drugs and co-delivery of two or more agents rendering multi-functionality [30-32].

Magnetic nanoparticles (MNPs) have drawn interest for their intrinsic magnetic property, which is advantageous for targeted drug delivery and biomedical imaging. MNPs lesser than $30 \mathrm{~nm}$ in diameter are superparamagnetic; a property that enables them to get temporarily magnetized in presence of a magnetic field and lose all magnetization once the field is removed. This property prevents unwanted agglomeration which is important for targeted delivery [33].

MNPs are also used as a contrast agent for magnetic resonance imaging (MRI) due to its ability to reduce the T2 relaxation time of water molecules $[34,35]$. More importantly, MNPs can be utilized for magnetic drug targeting (MDT), which is an active drug targeting method. During MDT, drugfunctionalized magnetic nanoparticles are administered into a body fluid, which can then be localized and concentrated at specific target sites using external magnetic fields. The active drug desorbs from the nanoparticles and initiates therapeutic activity. The most notable benefit of MDT is the ability to implement local drug action by providing a non-invasive, drug/ligand independent targeting technique. Although MNPs have been commonly administered via the blood stream, recent applications have targeted the CNS by intrathecal administration of MNPs into the subarachnoid space $[36,37]$.

Several research groups have developed MNPs for exploiting their properties as a contrast agents for MRI [38-42]. Other labs have accomplished site specific targeting of drug loaded MNPs using appropriate ligands, proteins and other molecules [43]. Enhanced permeation and retention effect aided in accumulating MNPs in target sites rather than relying on MDT [4446]. Despite these achievements, imaging MNPs via MRI during MDT remains difficult, because the magnetic fields used to generate images interfere with the magnetic fields needed for MDT. Therefore, there is a need for an MDT delivery vehicle that can be imaged in vivo without resorting to MRI.

In this paper, we present a nanovehicle that can be specifically targeted via MDT and have its distribution tracked during in vivo delivery. This vehicle comprises of a gold-coated magnetite nanoparticle $\left(\mathrm{Fe}_{3} \mathrm{O}_{4} @ \mathrm{Au}\right.$ NP) conjugated to cadmium telluride quantum dots (CdTe QDs). It offers three major benefits:

(i) The vehicle can be targeted, localized and concentrated at specific sites within tissues or organs; magnetite $\left(\mathrm{Fe}_{3} \mathrm{O}_{4}\right)$ core of these nanoparticles allows steering under the influence of externally placed magnets.

(ii) The outer gold shell of the vehicle can be loaded with therapeutic molecules. Drugs that have sulfur-containing functional groups show a strong affinity to bind to gold surfaces [47, 48], which enables conjugation of many active agents with relative ease.

(iii) The cadmium telluride quantum dots $(\mathrm{CdTe}$ QDs) can be imaged in vivo during delivery due to strong fluorescence emission.

Commercially available imaging modalities utilizing fluorescence molecular tomography (FMT) are capable of imaging this nanocomposite in vivo, due to the strong fluorescence emission by the quantum dots (QDs). QDs are nanometer sized highly fluorescent particles made of semiconductor materials, which do not photobleach easily. QDs retain their strong fluorescence even after conjugation to the nanoparticles, when compared to organic molecules such as rhodamine and fluorescein [49, 50]. QDs also have broader excitation spectra (high absorption) and a narrow, sharp emission peak [51], which results in a brighter emission and a higher signal to noise ratio.

This paper presents a novel QD-MNP drug delivery vehicle and is organized as follows. Methods section describes the synthesis and characterization results 
of our fluorescent nanocomposite and its individual components. The results section demonstrates cellular uptake, in vitro MDT and in vivo visualization of the nanocomposites. The paper closes with a discussion of our experimental results and conclusions.

\section{Materials and Methods}

The synthesis method of each of the individual components-gold coated magnetite nanoparticles ( $\left.\mathrm{Fe}_{3} \mathrm{O}_{4} @ \mathrm{Au} \mathrm{MNPs}\right)$, cadmium telluride quantum dots, and conjugation into a single nanocomposite has been described separately.

Sodium hydroxide $(\mathrm{NaOH})$ solution, ethanol (molecular biology grade) and 3-mercaptopropionic acid $\left(\mathrm{HSCH}_{2} \mathrm{CH}_{2} \mathrm{CO}_{2} \mathrm{H}\right)$ was purchased from Sigma Aldrich (MO, USA). 1 M TX-100 solution and trisodium citrate dihydrate $(\mathrm{HOC}(\mathrm{COONa})$ $\left.\left(\mathrm{CH}_{2} \mathrm{COONa}\right)_{2} \cdot 2 \mathrm{H}_{2} \mathrm{O}\right)$ was purchased from Fisher Scientific (NJ, USA). Hydrogen tetrachloroaurate (III) hydrate $\left(\mathrm{HAuCl}_{4}\right)$, sodium borohydride $\left(\mathrm{NaBH}_{4}\right)$ and sodium tellurite was purchased from Alfa Aesar (MA, USA). All other chemicals were purchased from Acros Organics (NJ, USA). Nanopure ${ }^{\circledR}$ water (Barnstead Nanopure lab water system) was used for all procedures described below.

This section starts with a detailed description for the synthesis of the magnetic nanocomposites $(\S 2.1, \S 2.2$ and $\S 2.3)$. Then cell-based experiments for proving biocompatibility and applicability of the nanocomposites in the CNS are described (\$2.4). An in vivo imaging experiment illustrates imaging ability of the vehicle. Finally, an MDT experiment demonstrates the magnetic targeting capability of our nanocomposites ( $\$ 2.5, \S 2.6$ and $\$ 2.7)$.

\section{Synthesis of GoldCoated Magnetite Nanoparticles}

The $\mathrm{Fe}_{3} \mathrm{O}_{4}$ cores were synthesized by a coprecipitation technique, well described by Mandal et al. [52], and various other articles, using ferrous and ferric salts to form $\mathrm{Fe}_{3} \mathrm{O}_{4}$ [53-55].

\section{Preparation of the Iron Stock Solution}

$1.543 \mathrm{~g}$ of ferric ammonium sulfate $\left[\mathrm{NH}_{4} \mathrm{Fe}\left(\mathrm{SO}_{4}\right)_{2} \cdot 12 \mathrm{H}_{2} \mathrm{O}\right]$ and $0.6275 \mathrm{~g}$ of ferrous ammonium sulfate $\left[\left(\mathrm{NH}_{4}\right)_{2} \mathrm{Fe}\left(\mathrm{SO}_{4}\right)_{2} \cdot 6 \mathrm{H}_{2} \mathrm{O}\right]$ were dissolved in $25 \mathrm{~mL}$ of $0.40 \mathrm{M}$ aqueous sulfuric acid solution to make the iron stock solution. The molar concentrations of the two iron salts in the solution were $0.128 \mathrm{M}$ and $0.064 \mathrm{M}$ respectively.

\section{Synthesis of Magnetite Nanoparticle Cores}

$1.5 \mathrm{~mL}$ of $1 \mathrm{M} \mathrm{TX}-100$ solution was added to $250 \mathrm{~mL}$ of $1 \mathrm{M} \mathrm{NaOH}$ solution and heated to $75^{\circ} \mathrm{C}$ in a water bath. $10 \mathrm{~mL}$ of the iron stock solution was added dropwise into this solution with vigorous nonmagnetic stirring. After complete addition of the stock solution, stirring was continued for another 20 minutes (min). During the entire procedure, the temperature was maintained at $75{ }^{\circ} \mathrm{C}$. Black $\mathrm{Fe}_{3} \mathrm{O}_{4}$ nanoparticle cores were obtained after settling followed by magnetic decantation to remove the supernatant. The particles were washed multiple times with water to remove the excess surfactant. The nanoparticles were then resuspended in water.

\section{Coating Magnetite Cores with Gold}

The solution containing the prepared $\mathrm{Fe}_{3} \mathrm{O}_{4}$ cores was sonicated for $15 \mathrm{~min}$ before placement in the water bath heated to $60{ }^{\circ} \mathrm{C}$. While slowly stirring the solution with a non-magnetic stirrer, $0.5 \mathrm{~g}$ of $\mathrm{D}(+)$ glucose was added. After $5 \mathrm{~min} 110 \mu \mathrm{L}$ of $1 \mathrm{~g} \mathrm{~mL}^{-1}$ aqueous solution of hydrogen tetrachloroaurate (III) hydrate was added dropwise. The temperature of the water bath was maintained at $60{ }^{\circ} \mathrm{C}$ and the solution was slowly stirred for $1 \mathrm{~h}$ in the dark. The color of the $\mathrm{Fe}_{3} \mathrm{O}_{4}$ nanoparticle cores changed to a brownred during the gold coating process. The solution was magnetically decanted and washed several times before being resuspended in water to a concentration of 0.17 $\mathrm{mg} \mathrm{mL} L^{-1}$.

\section{Synthesis of CdTe Quantum Dots}

The microwave based synthesis procedure was similar to Duan et al. [56] with modifications made to the microwaving procedure. The procedure yielded QDs in the 2-6 $\mathrm{nm}$ size range.

\section{Preparation of the cadmium chloride stock solution}

$0.4 \mathrm{~mL}$ of $0.04 \mathrm{M}$ of cadmium chloride solution was diluted with $42 \mathrm{~mL}$ of water to produce the stock solution.

\section{Preparation of the Cd-MPA-Te solution}

$100 \mathrm{mg}$ of trisodium citrate dihydrate, $4 \mathrm{~mL}$ of $0.01 \mathrm{M}$ sodium tellurite solution, $119 \mathrm{mg}$ of 3 mercaptopropionic acid and $50 \mathrm{mg}$ of sodium borohydride were added to the cadmium chloride stock 
solution under magnetic stirring. The molar ratio of Cd:MPA:Te in the solution was 1:7:0.25. The $\mathrm{pH}$ of the solution was maintained at 8 .

\section{Synthesis of the QDs by using microwaving technique}

$10 \mathrm{~mL}$ of the solution was placed in a $60 \mathrm{~mL}$ teflon digestion vessel (Savillex corporation, MN, USA). The QDs were synthesized with varying temperatures and microwaving time durations $\left(89^{\circ} \mathrm{C}-130{ }^{\circ} \mathrm{C}\right.$ and 1-5 $\mathrm{min})$ to produce QDs of different colors. The power of the microwave device (Samsung, $1.05 \mathrm{Kw}$ Micro Wave) was set at 900W. The QD samples were allowed to cool to room temperature prior to further examination.

\section{Synthesis of quantum dot conjugated gold coated magnetite nanocomposites (QDFe $\mathrm{O}_{4} @ \mathrm{Au}$ MNPs)}

$1 \mathrm{~mL}$ of both the $\mathrm{Fe}_{3} \mathrm{O}_{4} @ \mathrm{Au}$ MNPs and CdTe QD solutions $\left(0.17 \mathrm{mg} \mathrm{mL}^{-1}\right)$ was added to $20 \mathrm{~mL}$ of ethanol in a glass container. The glass container was kept in the dark for $48 \mathrm{~h}$ under constant slow stirring. This allowed for conjugation of the QDs to the gold coating of the $\mathrm{Fe}_{3} \mathrm{O}_{4} @ \mathrm{Au}$ MNPs to form the fluorescent nanocomposites.

The excess ethanol was removed post conjugation by magnetic decantation. To remove any excess QDs, the solution was washed with water to remove the free-floating water soluble QDs, followed by magnetic decantation to separate the nanocomposites. This process was repeated several times to remove all the free QDs from the solution, which can be confirmed by absence of fluorescence emission from the supernatant obtained on magnetic decantation. The washed nanocomposites are resuspended in pure water to obtain the desired concentration.

\section{Cell transfection experiment using $\mathbf{C 6}$ glioma cells}

In order to produce a cytotoxic effect in diseased target cells, nanocomposites need to be internalized. Therefore, the uptake of fluorescent nanocomposites into rat $\mathrm{C} 6$ glioma cells was studied in a transfection experiment. Cells were maintained in medium containing Dubelcco's Modified Eagle Medium (DMEM) and 10\% fetal bovine serum with $1 \%$ antibiotics. Various concentrations of the nanocomposites were prepared using the cell growth medium as diluent. $1 \mathrm{~mL}$ of this nanocomposite solution was applied to cells cultured in a 12-well plate. After incubation for 5 hours, cells were fixed with a $4 \%$ paraformaldehyde solution, while protecting it from light exposure to avoid any unwanted loss of fluorescence. Phalloidin-CruzFlour488 conjugate (Santa Cruz Biotechnology, 1:1000 solution in PBS) was applied onto the fixed sample for $60 \mathrm{~min}$. This was used to stain the cells' actin cytoskeleton. After rinsing phalloidin completely, DAPI (Biotium, 1:4000 solution in PBS) was applied for 5 min to stain chromatin in the nuclei of the cells. Cells were imaged using a normal Zeiss Axioskope fluorescence microscope.

The cellular uptake of the nanocomposites was quantified using Image $J$ software [57]. The quantification was performed by compiling three fluorescence images corresponding to the DAPI, nanocomposite and phalliodin stains (Fig. 4(a), 4(b), 4(c)). Phalloidin stains the actin cytoskeleton, marking the cell boundaries, and appears green; DAPI stains the nucleus, assisting in cell counting, and appears blue; and the nanocomposites appear red. Using the compiled image, only the portion of the image containing cells is selected and Image $J$ was used to quantify the average amount of red intensity per pixel. The average red intensity per pixel in the background of the composite image is subtracted from the average within the cell boundary. This final value provides a relative estimate of the nanocomposite uptake.

\section{Delivery of the nanocomposites into the intrathecal space of a Sprague Dawley rat}

12-week old Sprague Dawley rats were used for this procedure. All animal experiments were approved by the Animal Care and Use Committee at the University of Illinois at Chicago. The animals were anesthetized by inhalation of isoflurane in a closed chamber before being moved to a surgical table. The surgical table was equipped with stereotaxis equipment and a nose cone to allow for administration of gaseous isoflurane during surgery. Once anesthetized, the hair on the back of the animal was shaved using hair trimmers. This allowed for obtain better signal acquisition during the FMT procedure. An L3 laminectomy was performed and the dura bone was thinned down till it could be penetrated using a $30 \mathrm{G}$ needle. A small volume of 0.17 $\mathrm{mg} \mathrm{mL} \mathrm{m}^{-1}$ solution of the fluorescent nanocomposites was injected into the spinal subarachnoid space using a syringe pump. The imaging procedure described below 
was performed, afterwards rats were euthanized.

\section{In vivo imaging procedure}

FMT was performed using a Xenogen (Caliper Life Sciences) IVIS System equipped with anesthetic isoflurane system. The fluorescent nanocomposites required an excitation wavelength of $550 \mathrm{~nm}$ and the emission wavelength filter was set to $590 \mathrm{~nm}$. Other settings used for image acquisition were: binning level $=$ medium, fvalue $=\mathrm{f} / 2$ and acquisition time $=10$ seconds.

\section{MDT procedure}

To test the magnetic targeting capability of the nanocomposites, we developed a simple in vitro infusion system, in which amplitude and frequency of fluid pulsations can easily be adjusted. The model, as shown in Fig. 9, consists of a cylindrical polystyrene tube ( $49.0 \mathrm{~cm}$ length; $0.75 \mathrm{~cm}$ inner diameter) which encloses the fluid, which could be artificial CSF or water. A latex deformable membrane was tightly fixed to the mouth of the distal neck which expands and contracts to permit pulsatile motion of the fluid. Nanoparticle distribution inside the fluid varies depending on different patient specific parameters such as the frequency and magnitude of the pulsations, the velocity and stroke volume of fluid, and heart rate. In our lab, we are interested in the intrathecal delivery of the nanocomposites for treatment of CNS diseases. We therefore, imposed a fluid velocity of $1.5 \mathrm{~cm} \mathrm{~s}^{-1}$ and a stroke volume of $2 \mathrm{~mL}$, which are within the average ranges for CSF movement, as cited in prior literature $[58,59]$. Initially, we used water as the fluid in our system, because it has similar density to CSF [60].

To produce pulsatile fluid motion equal in amplitude and frequency to that of a typical resting human's CSF pulsations, the proximal end of the model was connected to a masterflex peristaltic pump (ColeParmer, IL, USA) and two of the three metal cylinders that drive the pump were removed. This created a ramping pulse function. Since CSF pulsates at a frequency of the heart beat, the pump was set at 70 beats per min which, according to the American Heart Association, is within the $60-80 \mathrm{bpm}$ range of normal resting heart rate in humans.

A predetermined volume of the nanocomposite solution $\left(0.17 \mathrm{~g} \mathrm{~mL}^{-1}\right)$ was injected into the model. A targeting magnet of $35 \mathrm{lb}$ pull force strength was placed at a distance of $10 \mathrm{~cm}$ from the injection site to collect and localize the injected nanocomposites. This region represents the targeting region. The experiment lasted for 10 min during which the accumulation at the target magnet site was imaged. No observable increase in the collection efficiency of nanocomposites at the targeting magnet site was observed beyond $10 \mathrm{~min}$.

\section{Results}

\section{Synthesis and Characterization results Gold-Coated Magnetite Nanoparticles}

The $\mathrm{Fe}_{3} \mathrm{O}_{4} @ \mathrm{Au}$ MNPs were characterized using transmission electron microscopy (TEM), energydispersive xray spectroscopy (EDS), selected area electron diffraction (SAED) and superconducting quantum interference device (SQUID) magnetometry. The $\mathrm{Fe}_{3} \mathrm{O}_{4} @ \mathrm{Au}$ MNPs used in our experiments had a $\mathrm{Fe}_{3} \mathrm{O}_{4}$ core with diameter between $812 \mathrm{~nm}$ (Fig. 1(a)) with a uniform gold shell thickness of $12 \mathrm{~nm}$, making the total diameter of the particle to be around 20-25 nm (Fig. 1(b)). $\mathrm{Fe}_{3} \mathrm{O}_{4}$ cores of this size exhibit superparamagnetic property retaining no net magnetization when removed from an external magnetic field [61]. They can be dispersed without agglomeration until an external magnetic field attracts the NPs, allowing for magnetic guidance and localization at the target site.

The gold coating on the $\mathrm{Fe}_{3} \mathrm{O}_{4}$ core (Fig. 1(c)) serves two purposes: (i) It prevents the oxidation of the $\mathrm{Fe}_{3} \mathrm{O}_{4}$ core into maghemite $\left(\mathrm{Fe}_{2} \mathrm{O}_{3}\right)$ by forming a protective coating layer. $\mathrm{Fe}_{2} \mathrm{O}_{3}$ has weaker magnetic susceptibility than $\mathrm{Fe}_{3} \mathrm{O}_{4}$ [62, 63], (ii) It also forms a convenient platform for loading drugs to the NP surface, since gold has a natural affinity for sulfur-containing functional groups and bonds.

EDS was used to confirm the chemical composition of the nanoparticles. The spectra obtained indicated the presence of the elements gold $(\mathrm{Au})$ and iron $(\mathrm{Fe})$. The SAED patterns of the $\mathrm{Fe}_{3} \mathrm{O}_{4}$ cores and $\mathrm{Fe}_{3} \mathrm{O}_{4} @$ $\mathrm{Au}$ MNPs indicate ring patterns confirming the face centered cubic structure of both $\mathrm{Fe}_{3} \mathrm{O}_{4}$ and $\mathrm{Au}$ respectively. In Fig. 2(a), the spacings measured in the diffraction patterns of $0.30 \mathrm{~nm}, 0.265 \mathrm{~nm}, 0.218$ $\mathrm{nm}$ and $0.150 \mathrm{~nm}$ are in agreement with the following standard reflections for $\mathrm{Fe}_{3} \mathrm{O}_{4}: 0.297 \mathrm{~nm}(220) ; 0.253$ (311); $0.210 \mathrm{~nm}$ (400); $0.148 \mathrm{~nm}$ (440). From the diffraction pattern shown in Fig. 2(b), the d-spacing is $0.236 \mathrm{~nm}$. This is close to the closed packed 
(a)
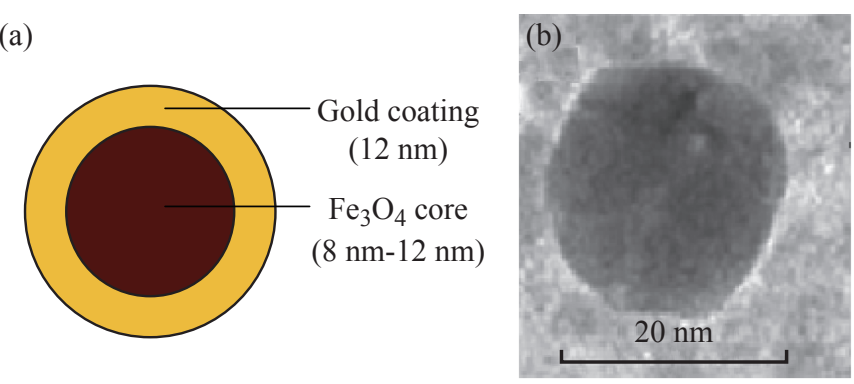

(c)

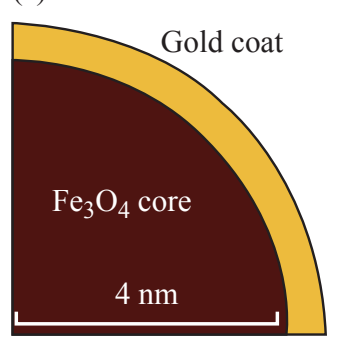

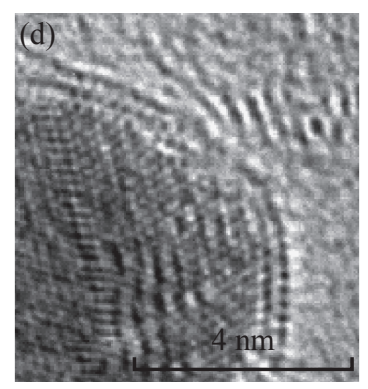
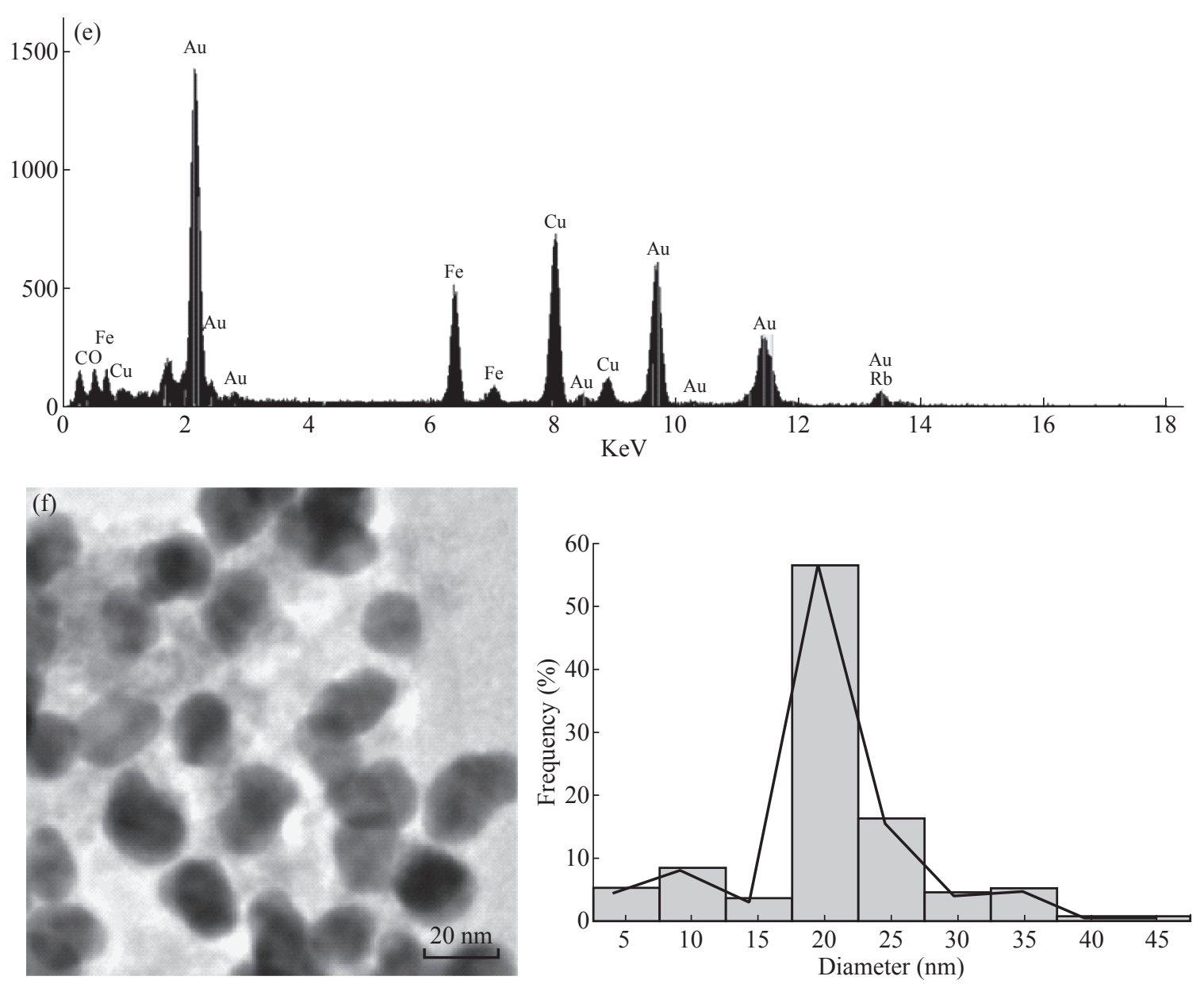

Fig. 1 Characterization of $\mathrm{Fe}_{3} \mathrm{O}_{4} @$ Au MNPs (a) Schematic of $\mathrm{Fe}_{3} \mathrm{O}_{4} @ \mathrm{Au}$ NP; (b) TEM image of $\mathrm{Fe}_{3} \mathrm{O}_{4} @$ au MNPs showing the total diameter of the particle to be between 20-25 nm; (c) Schematic of $\mathrm{Fe}_{3} \mathrm{O}_{4} @$ @ Au NP that has been partially coated with gold (d) TEM image (corresponding to schematic shown in Fig. 1(c)) that clearly delineates the $\mathrm{Fe}_{3} \mathrm{O}_{4}$ core and Au coating. This image confirms the length of core radius (4-6 nm); (e) EDS spectrum of $\mathrm{Fe}_{3} \mathrm{O}_{4} @ \mathrm{Au}$ MNPs which confirms the presence of elements Au, Fe and O; (f) TEM image showing multiple $\mathrm{Fe}_{3} \mathrm{O}_{4} @ \mathrm{Au}$ MNPs along with a histogram of their size distribution.

$\mathrm{Au}$ (111) spacing of $0.2355 \mathrm{~nm}$. These diffraction patterns confirm the $\mathrm{Fe}_{3} \mathrm{O}_{4}$ core and Au surface of our superparamagnetic nanoparticles. Determination of the magnetic properties of our nanoparticles was performed using SQUID magnetometry. From the plot of magnetization versus applied magnetic field (MH loop) at $265 \mathrm{~K}$ (Fig. 2(c)), the saturation magnetization $\left(M_{s}\right)$, remnant magnetization $\left(M_{r}\right)$, and coercivity $\left(H_{c}\right)$ for the $\mathrm{Fe}_{3} \mathrm{O}_{4} @ \mathrm{Au}$ MNPs were determined to be as follows: $M_{s}=29.5 \mathrm{emu} \mathrm{g}^{-1} ; M_{r}=0.52 \mathrm{emu} \mathrm{g}^{-1}$; $H_{c}=21.1 \mathrm{Oe}$ at $265 \mathrm{~K}$. The hysteresis loop indicates no remnant magnetization, establishing the desired superparamagnetism of the NPs.

\section{CdTe Quantum Dots}

A microwave irradiation procedure was used to produce fluorescent, stable, water soluble CdTe QDs, which served as the functional imaging entities of our drug delivery nanocomposite vehicle. Red QDs (2-3 $\mathrm{nm})$ were synthesized with the procedure described in section 2.2. QDs of varying colors can by synthesized by this technique. The colors arise from the quantum 

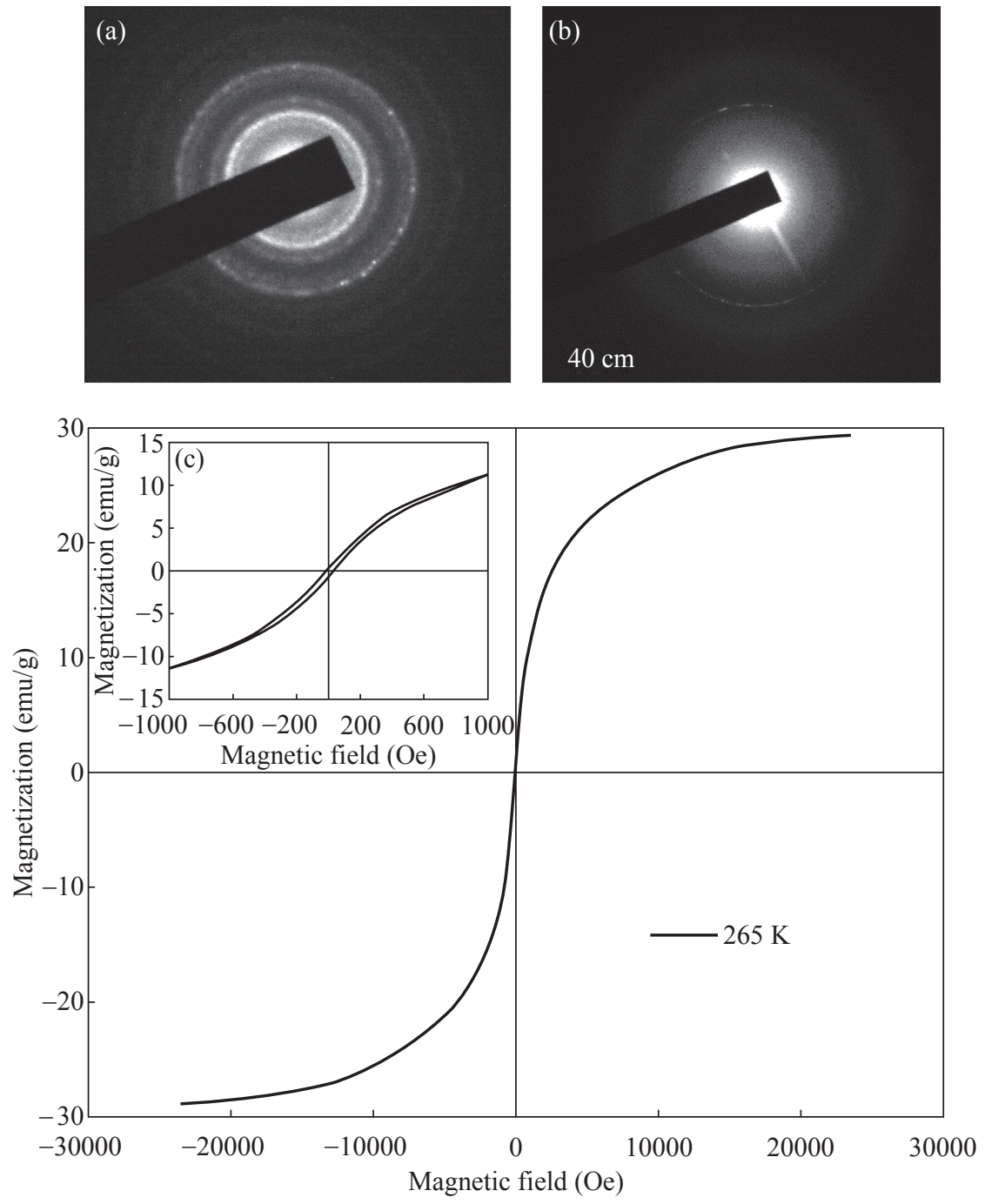

Fig. 2 Characterization of $\mathrm{Fe}_{3} \mathrm{O}_{4} @ \mathrm{Au}$ MNPs (a) SAED pattern of the $\mathrm{Fe}_{3} \mathrm{O}_{4}$ core; (b) SAED pattern of the $\mathrm{Fe}_{3} \mathrm{O}_{4} @ \mathrm{Au}$ MNPs; (c) M-H loop (magnetization versus applied magnetic field) obtained by SQUID magnetometry at $265 \mathrm{~K}$.

confinement effect since different QD radii produce different emission wavelengths $[51,64,65]$. Size tunability was observed and confirmed through HRTEM analysis (Fig. 3(a), 3(b)).

Fluorescence emission of the red QDs was confirmed using a fluorescence spectroscopy (Thermo Scientific NanoDrop 3300 fluorimeter). The absorption and fluorescence emission spectra of the QDs, shown in Fig. 3(d) and 3(e), indicate that the QDs have absorption and emission maxima peaks at $550 \mathrm{~nm}$ and $590 \mathrm{~nm}$ respectively. Red CdTe QDs were chosen for the synthesis of the nanocomposites because they have an emission peak of $590 \mathrm{~nm}$, which is better-suited for in vivo imaging.

\section{QD-Fe ${ }_{3} \mathrm{O}_{4} @ A u$ fluorescent nanocomposite}

The nanocomposites had a total diameter of around 30-45 nm, which consisted of a $\mathrm{Fe}_{3} \mathrm{O}_{4} @ \mathrm{Au} \mathrm{NP}$ core conjugated to QDs on its surface (Fig. 4(a)). These nanocomposites are superparamagnetic, because the $\mathrm{Fe}_{3} \mathrm{O}_{4} @ \mathrm{Au}$ MNPs that form the core of the structure are superparamagnetic. This property prevents agglomeration during delivery.

The stability of QDs after loading on the surface of the nanocomposites was studied with fluorescence and UV-Vis spectroscopy. When red QDs were loaded onto the MNP surface, the nanocomposites showed similar absorption and emission peaks as the plain red CdTe QDs, with the emission peak at $590 \mathrm{~nm}$. No change in the fluorescence spectral profile of the QD- $\mathrm{Fe}_{3} \mathrm{O}_{4} @ \mathrm{Au}$ nanocomposites was observed when compared to the plain QDs (Fig. 5(a)). FMT was also used to confirm the fluorescence emission of the conjugated structure in several environments, as shown in Table 1 and Fig. 4(d), 4(e). 

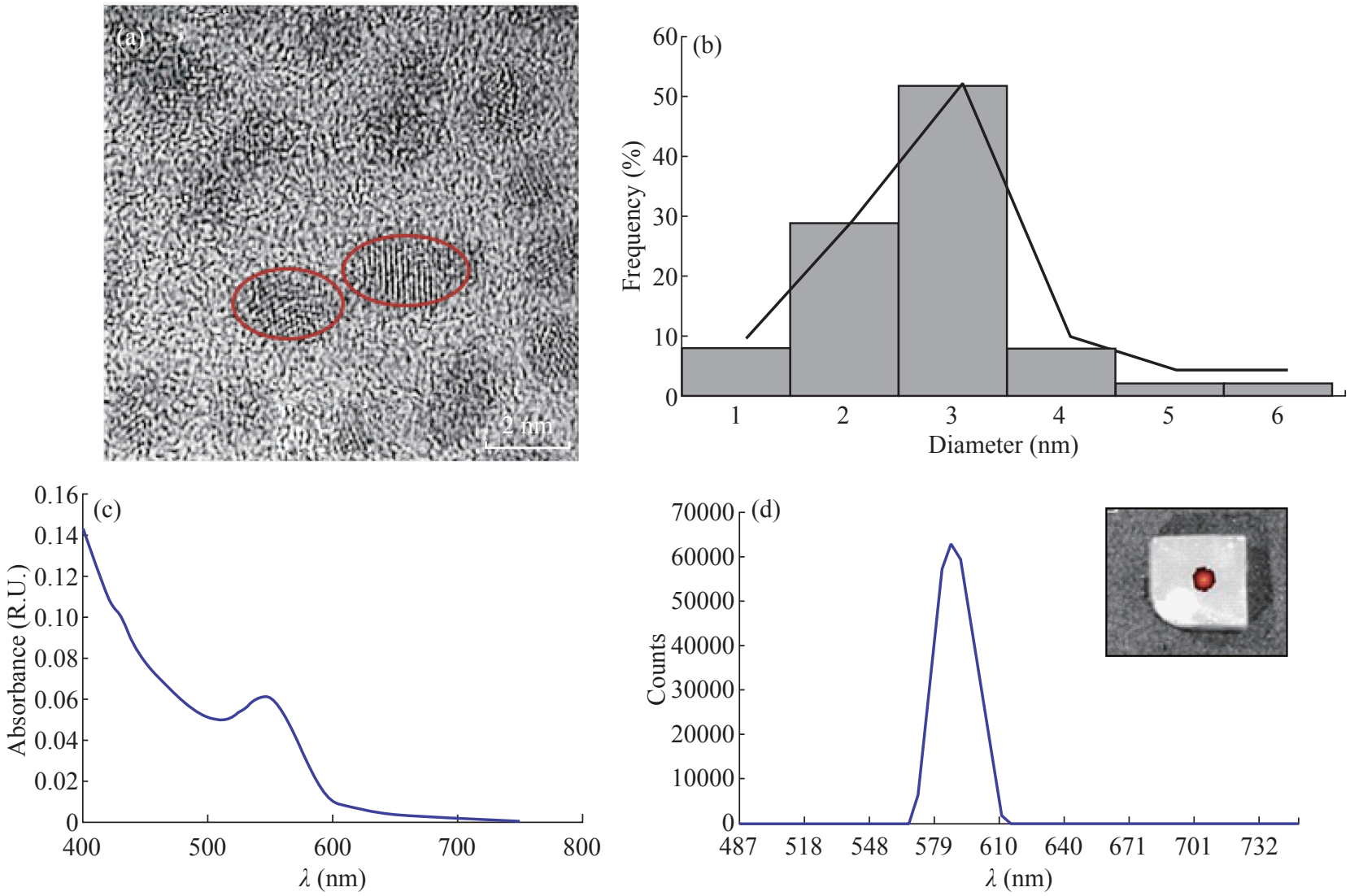

Fig. 3 Characterization of CdTe QDs (a) TEM image of red QDs measuring about 2-3 nm. Some of the QDs have been circled for better visibility; (b) Histogram showing the size distribution of the red QDs; (c) Absorption spectra of the CdTe QDs with a peak at $550 \mathrm{~nm}$; (d) Emission spectra of the CdTe QDs showing a peak at $590 \mathrm{~nm}$. An inset has been included which shows FMT image of QDs injected into a 6\% agarose gel.

In order to determine the fluorescence emission stability of the nanocomposites over an extended period of time, the nanocomposite solution was kept in the dark at room temperature and small samples were withdrawn at regular intervals to examine the fluorescence emission. A negligible loss of fluorescence was observed over a period of three weeks. After that time, fluorescence decayed exponentially (Fig. 5(b)), due to gradual oxidation of the CdTe QDs. Complete loss of fluorescence was observed after two months.

\section{Cellular Uptake Experiment}

Confocal microscopy images (Fig. 6(b)) indicate the presence of the QD- $\mathrm{Fe}_{3} \mathrm{O}_{4} @ \mathrm{Au}$ nanocomposites inside a C6 glioma cell. Insets B1 and B2 show z-stack images ( $\mathrm{x}-\mathrm{z}$ and $\mathrm{y}-\mathrm{z}$ respectively) of the cell confirming the presence of the nanocomposites inside it. Fig. 6(a) shows fluorescence images of the nanocomposites transfected into rat astrocyte cells.

The cell uptake experiment results (Fig. 7(a)) show that these nanocomposites are readily taken up by the cells. The C6 glioma cell uptake was quantified by using fluorescence measurements to understand the transfection of nanocomposites into the cells. A trend of increasing cellular uptake was observed as the incubation concentration of nanocomposites was increased (Fig. 7(a)).

\section{Enhancement of cellular uptake using magnetic fields}

Cell uptake of the nanocomposites in the presence of magnetic fields was studied. C6 glioma cells were incubated with a $0.3125 \mathrm{mg} \mathrm{mL}^{-1}$ solution of nanocomposites for $1 \mathrm{~h}$ in the presence of a magnetic field generated by a $3.23 \mathrm{lb}$ pull force permanent magnet. Fig. 7(b) shows a 5-fold increase in the nanocomposite uptake by the cells in the presence of the magnetic field $(\mathrm{N}=5)$. Similarly, a 300400\% increase in transfection was observed when solutions of $0.625 \mathrm{mg} \mathrm{mL}^{-1}, 1.25 \mathrm{mg} \mathrm{mL}^{-1}$, and $2.5 \mathrm{mg} \mathrm{mL}^{-1}$ concentrations were used in the presence of magnetic fields (data not shown). 

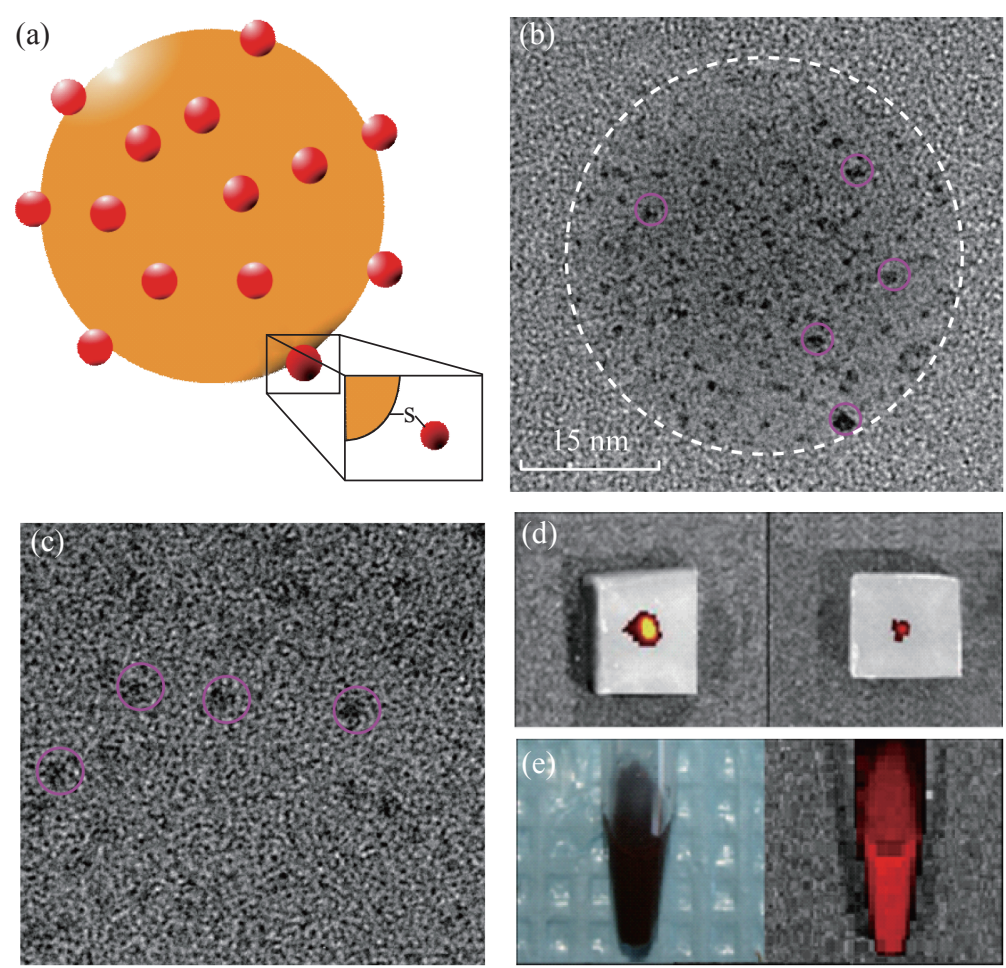

Fig. 4 Characterization of QD-Fe $\mathrm{O}_{4} @ \mathrm{Au}$ nanocomposites (a) Schematic of QD-Fe $\mathrm{O}_{4} @ \mathrm{Au}$ nanocomposite; (b) TEM image of the nanocomposite that shows both the nanocomposite core (white circle) as well as the conjugated QDs (smaller pink circles); (c) Enlarged view of the nanocomposite surface showing the individual QDs (pink circles); (d) FMT images of the QD-Fe $\mathrm{O}_{4} @ \mathrm{Au}$ nanocomposites injected in a $6 \%$ agarose gel; (e) Normal and FMT images of the nanocomposite solution.
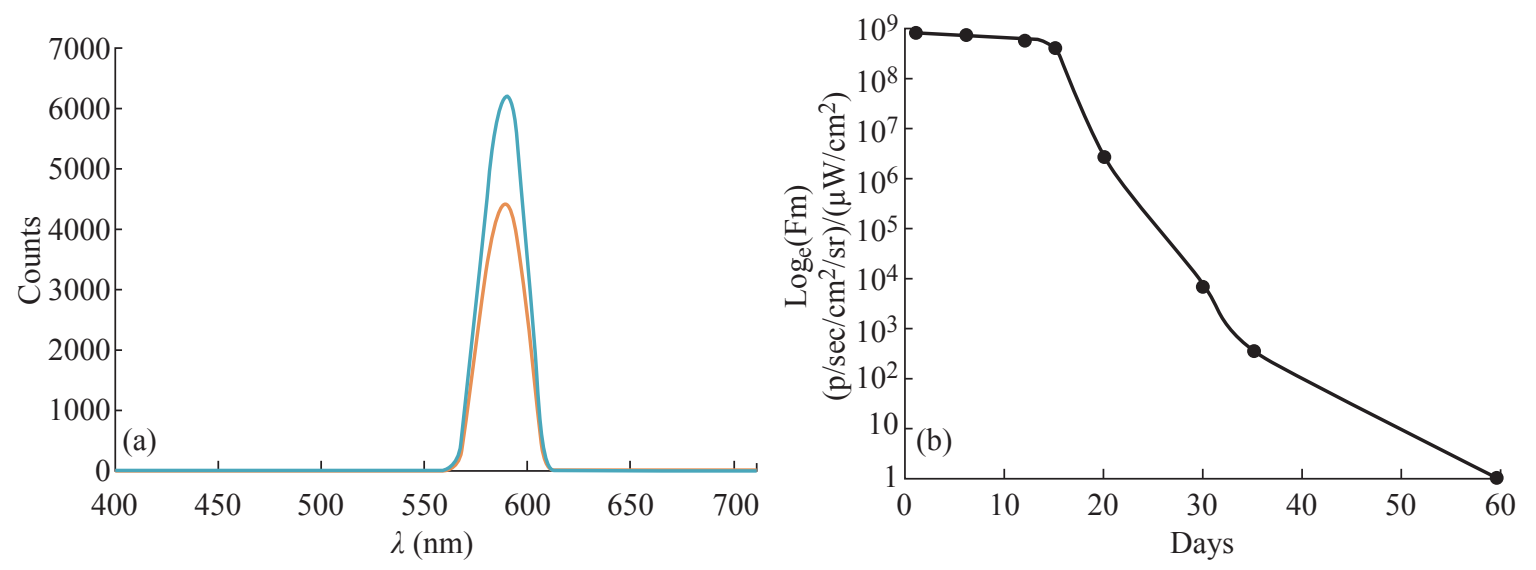

Fig. 5 Fluorescence characterization of QD-Fe $\mathrm{O}_{4} @$ @u nanocomposites (a) Emission spectra of the QD-Fe $\mathrm{O}_{4} @ \mathrm{Au}$ nanocomposites (orange) and plain CdTe QDs (blue). Maximum emission wavelengths for both were determined to be $590 \mathrm{~nm}$; (b) Graph showing the loss of fluorescence of the $\mathrm{QD}-\mathrm{Fe}_{3} \mathrm{O}_{4} @ \mathrm{Au}$ nanocomposites over time.

\section{Magnetic drug targeting}

MDT of our nanocomposites can be achieved by administration via the blood or CSF, both fluids move in a pulsatile manner in the body. To prove magnetic localization, the MDT experiment was conducted as mentioned in $\S 2.7$ to demonstrate magnetic targeting of our nanocomposites. It was repeated twice, with injection volumes of $1 \mathrm{~mL}$ and $0.5 \mathrm{~mL}$ of $0.17 \mathrm{~g} \mathrm{~mL}^{-1}$ nanocomposite solution, as shown in Fig. 8(b) and 8(c) respectively. Digital photo images were acquired every 2 min to observe the nanocomposite localization at the targeting magnet site. The QD- $\mathrm{Fe}_{3} \mathrm{O}_{4} @ \mathrm{Au}$ MNP solution can be observed with normal light due to its dark brown color. Both experiments indicate an increase in the accumulation of the nanocomposites with time, despite the mixing effect of the pulsatile motion of the fluid. The experiment was stopped at 

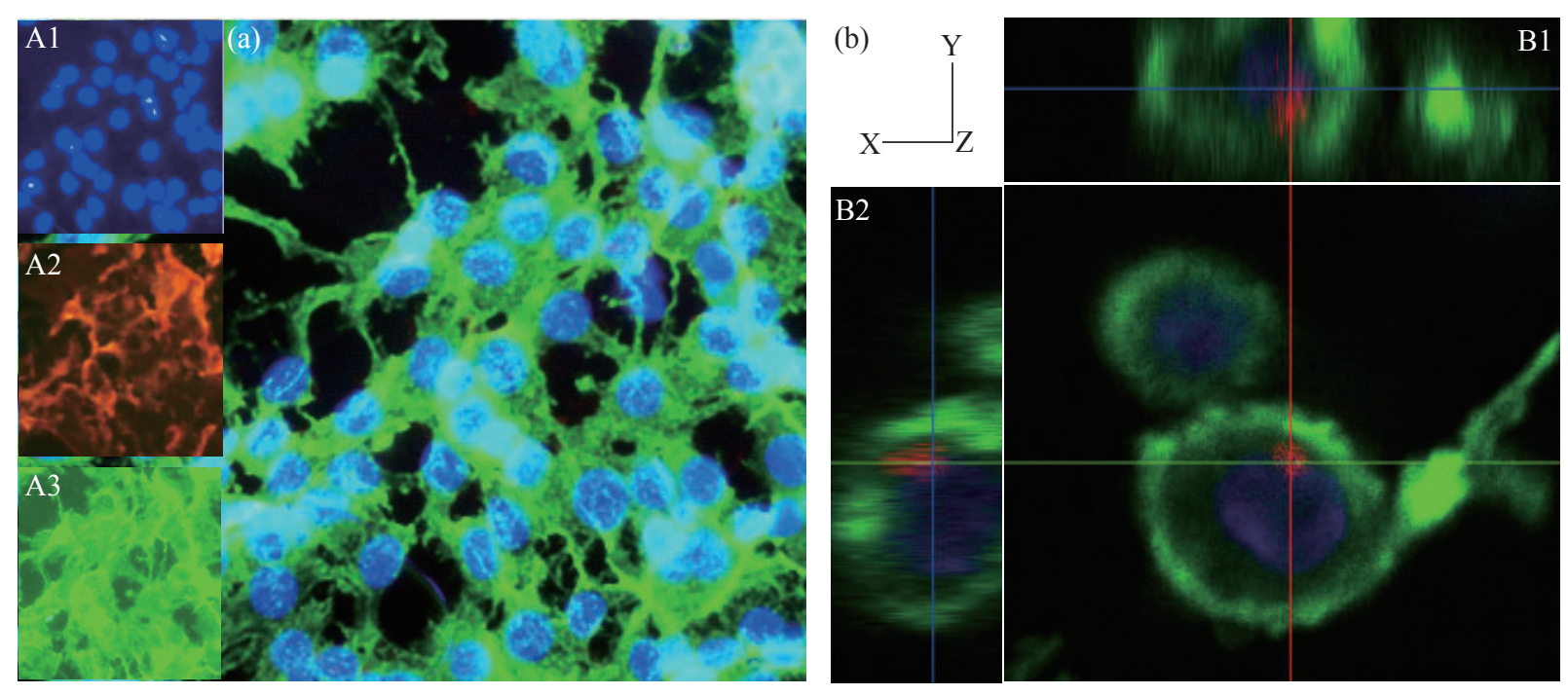

Fig. 6 Fluorescence microscopy results showing uptake by rat cortical astrocytes and C6 glioma cells on incubation in a $0.034 \mathrm{mg}$ $\mathrm{mL}^{-1} \mathrm{QD}-\mathrm{Fe}_{3} \mathrm{O}_{4} @ \mathrm{Au} \mathrm{NP}$ solution for $30 \mathrm{~min}$. All cells have been stained labeled using DAPI, which stains the nucleus blue, and phalloidin, which stains the actin cytoskeleton green. The nanocomposites appear red corresponding to the emission wavelength of $590 \mathrm{~nm}$. (a) Image showing nanocomposite localization within rat astrocyte cells. Insets contain images of separate stains: DAPI, QD-Fe $\mathrm{O}_{4} @ \mathrm{Au}$ MNPs and phalloidin; (b) Confocal microscopy images of QD-Fe $\mathrm{O}_{4} @$, $\mathrm{Au}$ MNPs transfected into C6 glioma cells. Insets $\mathrm{B} 1$ and $\mathrm{B} 2$ show the $\mathrm{z}$-stack images $(\mathrm{x}-\mathrm{z}$ and $\mathrm{y}-\mathrm{z})$ indicating the presence of nanocomposites inside the cell.
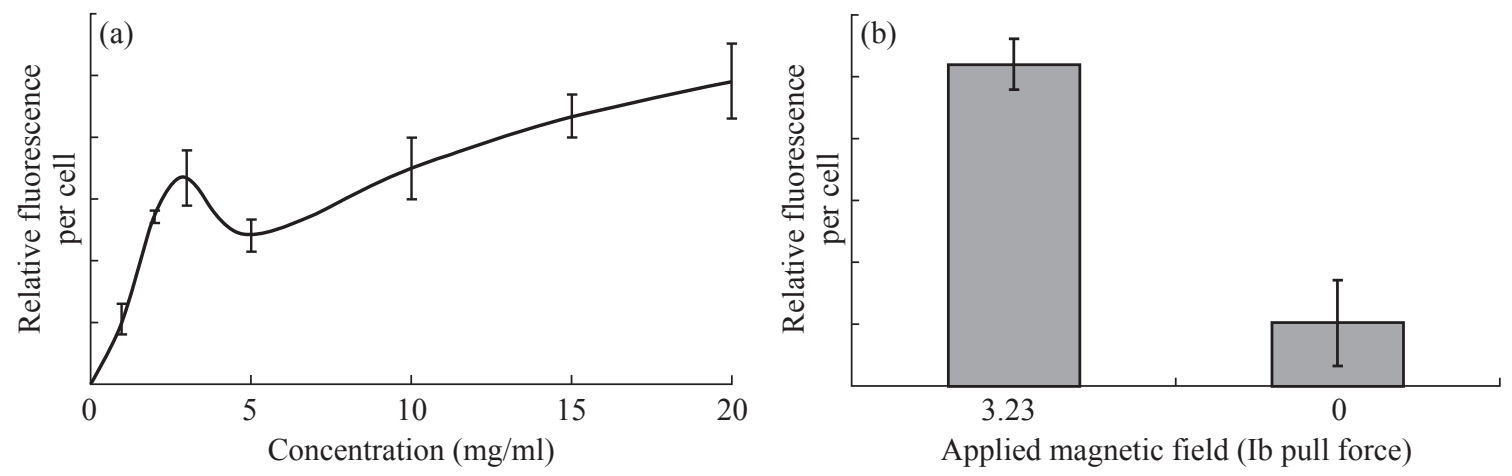

Fig. 7 Results of cell uptake and cytotoxicity studies using C6 glioma and rat cortical astrocyte cells (a) Fluorescence quantification of nanocomposite uptake in C6 glioma cells. A spike in nanocomposite uptake is observed at a lower concentration of nanocomposite solution $\left(2.5 \mathrm{mg} \mathrm{mL}^{-1}\right)$. (b) Uptake of nanocomposites $\left(0.3125 \mathrm{mg} \mathrm{mL}^{-1}\right)$ in $\mathrm{C} 6$ glioma cells under the influence of a magnetic field. Increased transfection occurs under the influence of a $3.23 \mathrm{lb}$ pull force magnetic field.

the 10 min time point, because no further increase in nanocomposite collection was observed. Maximum accumulation occurred at the edges of the magnet, as the field strength is the greatest at this region. Almost no nanocomposites were found to cross the magnet and move towards the peristaltic pump, as can be seen in frame 6 of Fig. 8(b).

Such a barrier created by the magnetic field is very useful for drug delivery treatments where a cytotoxic drug should be confined to a target zone, such as a spinal tumor, without spreading to other regions. During both experiments, at least $80 \%$ of the nanocomposites were collected at the target magnet site, with larger accumulation observed for the smaller injection volume. Magnet size, shape and field strength can be modulated depending on required drug dosage for therapeutic efficacy and patient convenience.

\section{In vivo imaging results}

To demonstrate live imaging capability, the fluorescence emission of these nanocomposites was measured through both rat muscle and bone tissue. $200 \mu \mathrm{L}$ of the nanocomposite solution $\left(2.5 \mathrm{mg} \mathrm{mL}^{-1}\right)$ was injected into the abdominal muscle of a Sprague Dawley rat at a depth of $1 \mathrm{~cm}$ from the skin surface. FMT images were generated using the IVIS ${ }^{\circledR}$ system. Fig. 9(a), 9(b) shows that the nanocomposites are clearly visible, demonstrating that deep tissue imaging 


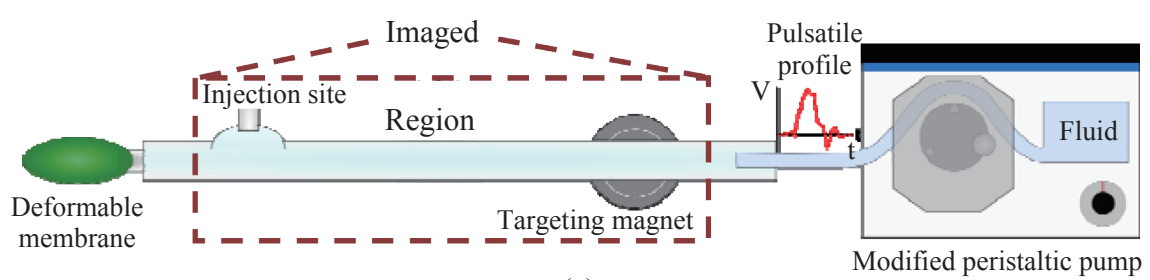

(a)
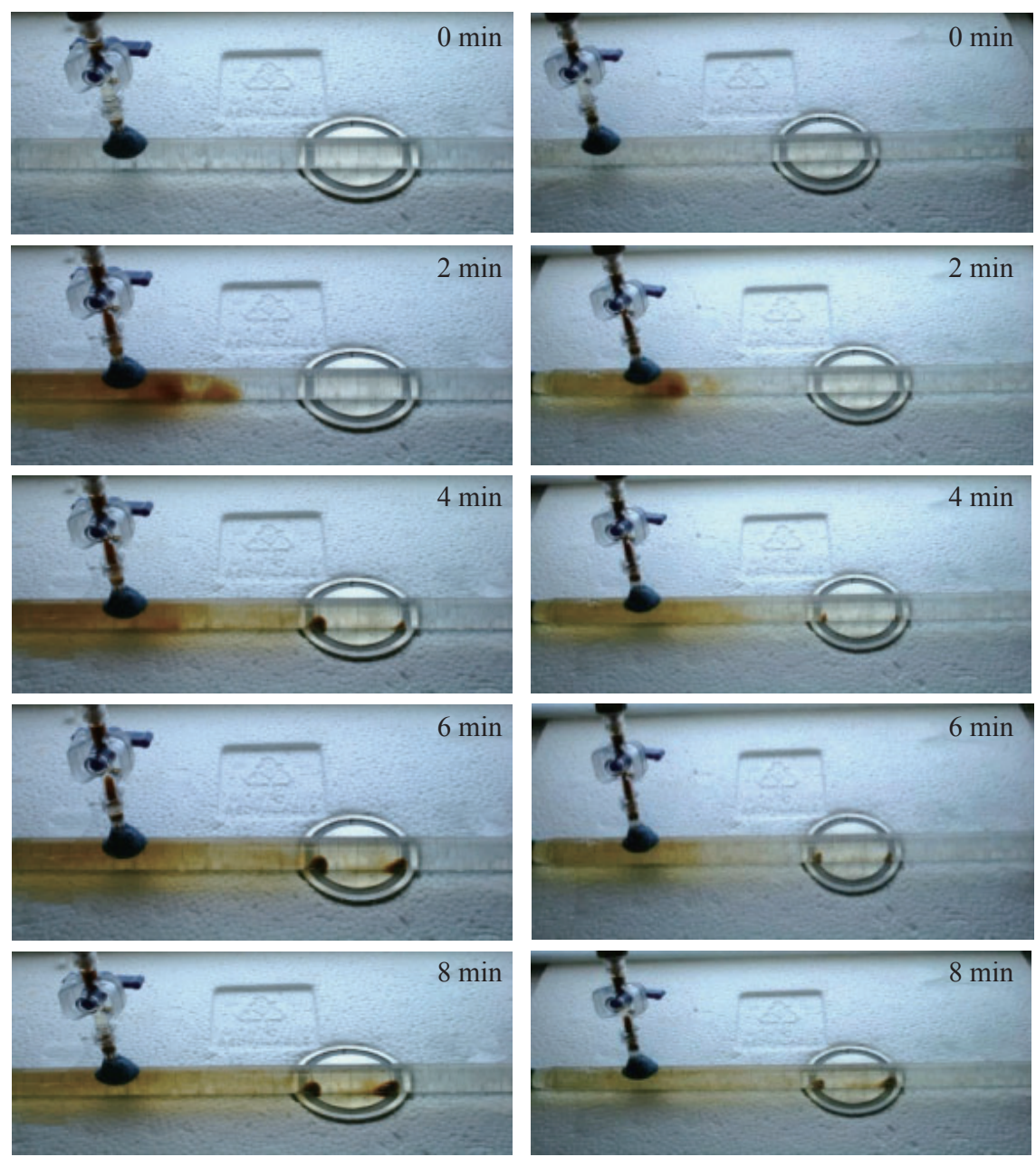

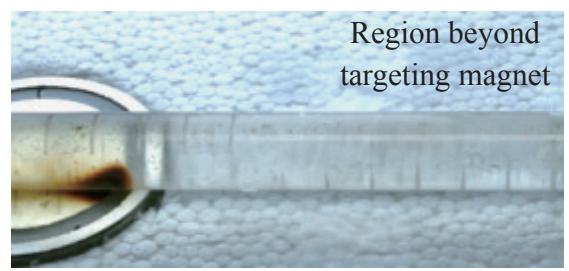

(b)

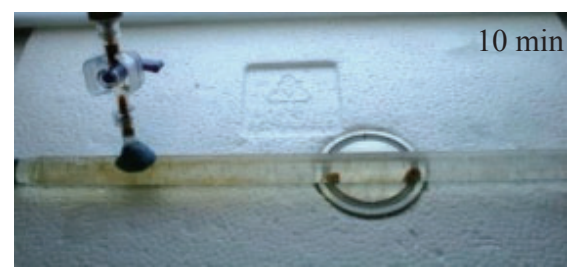

(c)

Fig. 8 In vitro magnetic drug targeting experiment (a) Schematic of the experimental setup containing the injection site, targeting magnet and peristaltic pump to create the pulsatile motion of the fluid; (b) (c) Images of the MDT experiment showing localization of the nanocomposites at the target site for an injection volume of (b) $1 \mathrm{~mL}$ and (c) $0.5 \mathrm{~mL}$.

is feasible using this drug delivery vehicle.

The BBB creates a great obstacle for effective drug delivery to the CNS $[66,67]$. To show the ability to track our drug delivery vehicle inside the CNS of animals, these particles were intrathecally injected into the lumbar region of a rat. Fig. 9(b), 9(c) shows the images generated from the IVIS ${ }^{\circledR}$ system when small volumes of the nanocomposites were injected into the spinal subarachnoid space (at the L3 region) of the rat. The fluorescence maps indicate that visualization is possible through bone and tissue of the spine. The fluorescence emission of the nanocomposites in all 

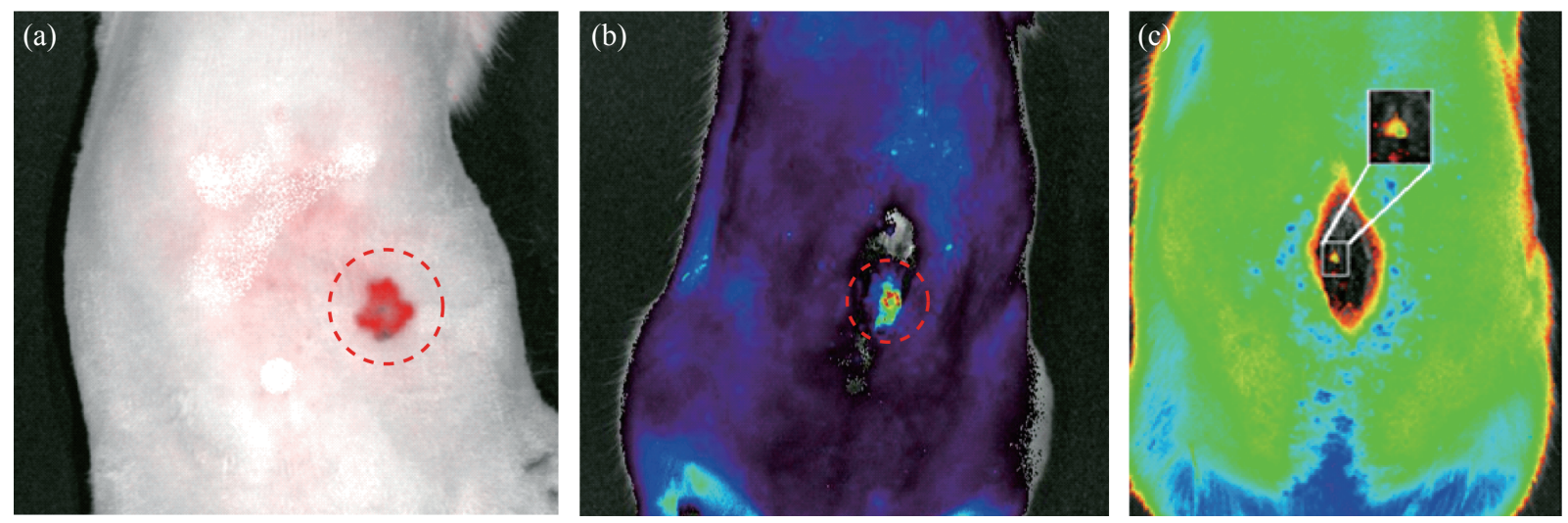

Fig. 9 In vitro imaging of our nanocomposites using IVIS® system in a Sprague Dawley rat. (a) FMT image of the ${ }^{\circledR}$ D-Fe $\mathrm{O}_{4} @$ Au nanocomposites $(200 \mu \mathrm{L})$ injected into the abdominal muscle of a rat at a depth of $1 \mathrm{~cm}$; FMT image of the QD-Fe $\mathrm{O}_{4} @ \mathrm{Au}$ nanocomposites injected into the intrathecal space of a Sprague Dawley rat (c) Injection volume $=200 \mu \mathrm{L}$; (d) Injection volume $=50$ $\mu \mathrm{L}$.

Table 1 Epifluorescence measurements made from the nanocomposites in different environments

\begin{tabular}{cc}
\hline Sample Environment & Epifluorescence generated $\left(\mathrm{p} \mathrm{sec}^{-1} \mathrm{~cm}^{-2} \mathrm{sr}^{-1}\right) /\left(\mu \mathrm{W} \mathrm{cm}{ }^{-2}\right)$ \\
\hline$\left(\mathrm{QD}-\mathrm{Fe}_{3} \mathrm{O}_{4} @\right.$ Au nanocomposites at a concentration of $2.5 \mathrm{mg} \mathrm{mL}$ & $7.68 \times 108$ \\
\hline $6 \%$ agarose gel $(\mathrm{depth}=0.25 \mathrm{~cm})$ & $6.02 \times 108$ \\
$6 \%$ agarose gel $($ depth $=0.5 \mathrm{~cm})$ & $5.82 \times 108$ \\
In vivo (depth $=1 \mathrm{~cm})$ : rat flesh & $2.50 \times 107$ \\
In vivo (depth $=0.3 \mathrm{~cm})$ : intrathecal space & \\
\hline
\end{tabular}

environments, especially in in vivo environments is significant, indicating its applicability for in vivo drug delivery purposes, as shown in Table 1.

\section{Discussion}

Our results show the successful synthesis and characterization of a novel drug delivery vehicle for MDT that can be visualized in vivo during delivery to the targeted disease site. The magnetic core of the nanocomposite is superparamagnetic, therefore, targeting drugs using this vehicle via MDT is possible. External magnetic fields can arrest the nanocomposites within any targeted region, despite the convective transport and mixing effect caused by the pulsatile motion of CSF as shown by the in vitro MDT experiment. Moreover, the cellular uptake experiments demonstrated internalization of nanocomposites in tumor cells. The vehicle was successfully visualized various environments (cellular, in vitro and in vivo) using FMT techniques, offered by commercial imaging equipment such as the IVIS ${ }^{\circledR}$ system (Caliper Life Sciences).

All the necessary attributes for an MDT drug delivery vehicle have been attained in our nanocomposites, such as magnetic guidability and in vivo imageability. Drug functionalization can also be achieved due to the affinity that gold has for sulfurcontaining functional groups and bonds (e.g. thiol and mercapto bonds), which are found in many proteins, pharmaceutical and biopharmaceutical drugs. Unlike previous studies which suffered fluorescence quenching after gold surfaces conjugation [68, 69], our QD conjugation retained strong fluorescence emission even after conjugation to the gold-coated magnetic nanoparticle. Fluorescence emission from our nanocomposite structure was stable over a three-week period of time, demonstrating its suitability for longer term experiments.

The gold and $\mathrm{Fe}_{3} \mathrm{O}_{4}$ of our nanocomposites are biocompatible. $\mathrm{Fe}_{3} \mathrm{O}_{4}$ degrades into its components and can be integrated in normal iron homeostasis, without posing a threat $[70,71]$. A disadvantage of our system is that the CdTe QDs are not biocompatible. It was observed that concentrations of upto $20 \mathrm{mg} / \mathrm{mL}$ of our nanocomposites containing CdTe QDs were taken up by cells over a period of $5 \mathrm{~h}$. However, higher concentrations would lead to toxic effects. One of the key steps in our future work will be to synthesize CdTe QDs with a shell of another compound such as $\mathrm{ZnS}$ to prevent release of $\mathrm{Cd}^{2+}$ ions, which has a detrimental effect on cells [72]. Our CdTe QDs 
capped with thiol bonds are synthesized using 3 mercaptopropionic acid. Another possibility would be to use a different stabilizer during the synthesis process, such as tripeptide glutathione (GSH) instead of the 3 mercaptopropionic acid. This would results in biocompatible CdTe QDs capped with GSH group [73]. The GSH group contains a sulfur atom, and QDs capped with GSH can be loaded on the gold surface of our nanocomposite using a similar technique as mentioned in $\S 2.3$. GSH can be used to detoxify $\mathrm{Cd}^{2+}$ ions in medicine due to its chelating capability [74].

We believe that for MDT, the drug dosages used would be much lower compared to systemic delivery, as the therapeutic molecules can be specifically delivered at target sites. Therefore, a very high concentration of our nanocomposites would not be used for drug delivery. Also, in vivo studies suggest that, regardless of the nature of the QDs, vertebrate systems tend to recognize them as foreign, with rapid elimination of the materials through the primary excretory-organs/ systems: the liver, spleen, and lymphatic systems [75]. Once inside the target cell, gold-thiol bonds are rapidly broken due to the reducing environment of the cell cytoplasm, and the QDs will be released from the $\mathrm{Fe}_{3} \mathrm{O}_{4} @ \mathrm{Au}$ NP core. QDs of sizes similar to the ones that we use can be easily and efficiently cleared from the body [75]. Specific biocompatibility studies would be needed for the definite concentration ranges that are safe for therapeutic application.

\section{Conclusions}

This nanovehicle has possible MDT applications for the treatment of gliomas, spasticity, pain and other diseases where focal targeting and in vivo visualization are required. It can also be applied in CNS disease treatments where guidance to the target site has proven to be a major challenge. MDT is an effective technique to achieve localized drug action without systemic toxicity within the CNS.

Future work would involve increasing the biocompatibility of our nanocomposites by utilizing techniques such as synthesis of core-shell QDs that contain an inner core of CdTe and an outer shell of a more biocompatible material such as ZnS or ZnTe. Our future research would also involve conjugating drug molecules to the nanocomposite surface followed by in vivo validation of MDT using an animal model. Such multifunctional nanoparticles hold great promise for targeted disease treatment.

\section{Acknowledgment}

TF and DA would like to acknowledge their fellowships from the National Science FoundationResearch Experience for Teachers (NSF-RET) Program.

\section{Funding Sources}

This work was supported in part by the National Science Foundation Grant-CBET 1010621 and the NSF RET EEC 1132694.

\section{References}

[1] J.A. DiMasi, R.W. Hansen, H.G. Grabowski, et al., Cost of innovation in the pharmaceutical industry. J. Health Econ., 1991, 10(2): 107-142.

[2] L.B. Cardinal, Technological Innovation in the Pharmaceutical Industry: The Use of Organizational Control in Managing Research and Development. Organ. Sci., 2001, 12(1): 19-36.

[3] T.K. Jain, J. Richey, M. Strand, et al., Magnetic nanoparticles with dual functional properties: Drug delivery and magnetic resonance imaging. Biomaterials, 2008, 29(29): 4012-4021.

[4] N. Kohler, C. Sun, A. Fichtenholtz, et al., MethotrexateImmobilized Poly(ethylene glycol) Magnetic Nanoparticles for MR Imaging and Drug Delivery. Small, 2006, 2(6): 785-792.

[5] S. Mornet, J. Portier, E. Duguet, A method for synthesis and functionalization of ultrasmall superparamagnetic covalent carriers based on maghemite and dextran. $J$. Magn. Magn. Mater., 2005, 293(1): 127-134.

[6] M. Liong, J. Lu, M. Kovochich, et al., Multifunctional Inorganic Nanoparticles for Imaging, Targeting, and Drug Delivery. ACS Nano., 2008, 2(5): 889-896.

[7] J.R. McCarthy, R. Weissleder, Multifunctional magnetic nanoparticles for targeted imaging and therapy. Adv. Drug Deliv. Rev., 2008, 60(11): 1241-1251.

[8] N. Sanvicens, M.P. Marco, Multifunctional nanoparticles properties and prospects for their use in human medicine. Trends Biotechnol., 2008, 26(8): 425-433.

[9] B.J. Boyd, Past and future evolution in colloidal drug delivery systems. Expert Opin. Drug Deliv., 2008, 5(1): 69-85.

[10] C. Sun, J.S.H. Lee, M. Zhang, Magnetic nanoparticles in MR imaging and drug delivery. Adv. Drug Deliv. Rev., 2008, 60(11): 1252-1265.

[11] R.D.K. Misra, Magnetic nanoparticle carrier for targeted drug delivery: perspective, outlook and design. Mater. Sci. Technol., 2008, 24(9): 1011-1019.

[12] J.R. McCarthy, K.A. Kelly, E.Y. Sun, et al., Targeted delivery of multifunctional magnetic nanoparticles. Nanomed., 2007, 2(2): 153-167.

[13] D. Kim, Y.Y. Jeong, S. Jon, A Drug-Loaded Aptamer - Gold Nanoparticle Bioconjugate for Combined CT Imaging and Therapy of Prostate Cancer. ACS Nano, 2010, 4(7): 3689-3696.

[14] M.E. Davis, Non-viral gene delivery systems. Curr. Opin. 
Biotechnol., 2002, 13(2): 128-131.

[15] J.M. Harris, R.B. Chess, Effect of pegylation on pharmaceuticals. Nat. Rev. Drug Discov., 2003, 2(3): 214221.

[16] B. Duncan, C. Kim, V.M. Rotello, Gold nanoparticle platforms as drug and biomacromolecule delivery systems. J. Controlled Release, 2010, 148(1): 122-127.

[17] G. Han, P. Ghosh, V.M. Rotello, Functionalized gold nanoparticles for drug delivery. Nanomed., 2007, 2(1): 113-123.

[18] S.D. Brown, P. Nativo, J.A. Smith, et al., Gold Nanoparticles for the Improved Anticancer Drug Delivery of the Active Component of Oxaliplatin. J. Am. Chem. Soc., 2010, 132(13): 4678-4684.

[19] H.D.M. Hettiarachchi, Y. Hsu, T.J.H. Jr, et al., The Effect of Pulsatile Flow on Intrathecal Drug Delivery in the Spinal Canal. Ann. Biomed. Eng., 2011, 39(10): 25922602.

[20] W.M Pardridge, Brain drug targeting: the future of brain drug development. Cambridge; New York: Cambridge University Press; 2001

[21] G.R. Reddy, M.S. Bhojani, P. McConville, et al., Vascular Targeted Nanoparticles for Imaging and Treatment of Brain Tumors. Clin. Cancer Res., 2006, 12(22): $6677-$ 6686.

[22] W.S. Enochs, G. Harsh, F. Hochberg, et al., Improved delineation of human brain tumors on MR images using a long-circulating, superparamagnetic iron oxide agent. $J$. Magn. Reson. Imaging, 1999, 9(2): 228-232.

[23] M.E. Davis, Z (Georgia) Chen, D.M. Shin, Nanoparticle therapeutics: an emerging treatment modality for cancer. Nat. Rev. Drug Discov., 2008, 7(9): 771-782.

[24] O. Veiseh, J.W. Gunn, M. Zhang, Design and fabrication of magnetic nanoparticles for targeted drug delivery and imaging. Adv. Drug Deliv. Rev., 2010, 62(3): 284-304.

[25] D.A. Gorin, S.A. Portnov, O.A. Inozemtseva, et al., Magnetic/gold nanoparticle functionalized biocompatible microcapsules with sensitivity to laser irradiation. Phys. Chem. Chem. Phys., 2008, 10(45): 6899.

[26] Q. He, J. Shi, Mesoporous silica nanoparticle based nano drug delivery systems: synthesis, controlled drug release and delivery, pharmacokinetics and biocompatibility. $J$. Mater. Chem., 2011, 21(16): 5845.

[27] J.E. Kipp, The role of solid nanoparticle technology in the parenteral delivery of poorly water-soluble drugs. Int. J. Pharm., 2004, 284(1-2): 109-122.

[28] J. Zhang, R.D.K. Misra, Magnetic drug-targeting carrier encapsulated with thermosensitive smart polymer: Coreshell nanoparticle carrier and drug release response. Acta Biomater, 2007, 3(6): 838-850.

[29] G. Huang, J. Gao, Z. Hu, et al., Controlled drug release from hydrogel nanoparticle networks. J. Controlled Release, 2004, 94(2-3): 303-311.

[30] J. Hu, D.K.P. Johnston, R. O. Williams 3rd, Nanoparticle Engineering Processes for Enhancing the Dissolution Rates of Poorly Water Soluble Drugs., 2004, 3:233-245.

[31] Y. Wang, S. Gao, W.H. Ye, et al., Co-delivery of drugs and DNA from cationic core-shell nanoparticles selfassembled from a biodegradable copolymer. Nat. Mater., 2006, 5(10): 791-796.

[32] L. Zhang, A.F. Radovic-Moreno, F Alexis, et al., CoDelivery of Hydrophobic and Hydrophilic Drugs from Nanoparticle-Aptamer Bioconjugates. Chem. Med. Chem., 2007, 2(9): 1268-1271.

[33] A. Jordan, R. Scholz, K Maier-Hauff, et al., Presentation of a new magnetic field therapy system for the treatment of human solid tumors with magnetic fluid hyperthermia. J. Magn. Magn. Mater., 2001, 225(1): 118-126.

[34] H.B. Na, I.C. Song, T. Hyeon, Inorganic Nanoparticles for MRI Contrast Agents. Adv. Mater, 2009, 21(21): 2133-
2148.

[35] S.A. Corr, S.J. Byrne, R. Tekoriute, et al., Linear Assemblies of Magnetic Nanoparticles as MRI Contrast Agents. J. Am. Chem. Soc., 2008, 130(13): 4214-4215.

[36] E. Lueshen, I. Venugopal, J. Kanikunnel, et al., Intrathecal magnetic drug targeting using gold-coated magnetite nanoparticles in a human spine model. Nanomed., 2014, 9: 1155-69

[37] E. Lueshen, I. Venugopal, T. Soni, et al., Implant-Assisted Intrathecal Magnetic Drug Targeting to Aid in Therapeutic Nanoparticle Localization for Potential Treatment of Central Nervous System Disorders. J. Biomed. Nanotechnol., 2015, 11: 253-61

[38] J. Kim, H.S. Kim, N. Lee, et al., Multifunctional Uniform Nanoparticles Composed of a Magnetite Nanocrystal Core and a Mesoporous Silica Shell for Magnetic Resonance and Fluorescence Imaging and for Drug Delivery. Angew. Chem. Int. Ed., 2008, 47(44): 8438-8441.

[39] N. Nasongkla, E. Bey, J. Ren, et al., Multifunctional Polymeric Micelles as Cancer-Targeted, MRIUltrasensitive Drug Delivery Systems. Nano Lett., 2006, 6(11): 2427-2430.

[40] J. Kim, J.E. Lee, S.H. Lee, et al., Designed Fabrication of a Multifunctional Polymer Nanomedical Platform for Simultaneous Cancer- Targeted Imaging and Magnetically Guided Drug Delivery. Adv. Mater., 2008, 20(3): 478-483.

[41] J.E. Lee, N. Lee, H. Kim, et al., Uniform Mesoporous Dye-Doped Silica Nanoparticles Decorated with Multiple Magnetite Nanocrystals for Simultaneous Enhanced Magnetic Resonance Imaging, Fluorescence Imaging, and Drug Delivery. J. Am. Chem. Soc., 2010, 132(2): 552-557.

[42] J.H. Park, G von Maltzahn, E. Ruoslahti, et al., Micellar Hybrid Nanoparticles for Simultaneous Magnetofluorescent Imaging and Drug Delivery. Angew. Chem., 2008, 120(38): 7394-7398.

[43] X. Yang, H. Hong, J.J. Grailer, et al., cRGDfunctionalized, DOX-conjugated, and $64 \mathrm{Cu}$-labeled superparamagnetic iron oxide nanoparticles for targeted anticancer drug delivery and PET/MR imaging. Biomaterials, 2011, 32(17): 4151-4160.

[44] M.K. Yu, Y.Y. Jeong, J. Park, et al., Drug-Loaded Superparamagnetic Iron Oxide Nanoparticles for Combined Cancer Imaging and Therapy In Vivo. Angew. Chem. Int. Ed., 2008, 47(29): 5362-5365.

[45] B. Chertok, B.A. Moffat, A.E. David, et al., Iron oxide nanoparticles as a drug delivery vehicle for MRI monitored magnetic targeting of brain tumors. Biomaterials, 2008, 29(4): 487-496.

[46] F. Gazeau, M. Lévy, C. Wilhelm, Optimizing magnetic nanoparticle design for nanothermotherapy. Nanomed., 2008, 3(6): 831-844.

[47] M.C. Bourg, A. Badia, R.B. Lennox, Gold-Sulfur Bonding in 2D and 3D Self-Assembled Monolayers: XPS Characterization. J. Phys. Chem. B, 2000, 104(28): $6562-$ 6567.

[48] S.H. Lee, K.H. Bae, S.H. Kim, K.R. Lee, T.G. Park, Amine-functionalized gold nanoparticles as non-cytotoxic and efficient intracellular siRNA delivery carriers. Int. $J$. Pharm., 2008, 364(1): 94-101.

[49] W.C.W. Chan, S. Nie, Quantum Dot Bioconjugates for Ultrasensitive Nonisotopic Detection. Science, 1998, 281(5385): 2016-2018.

[50] Z. Kaul, T. Yaguchi, S.C. Kaul, et al., Mortalin imaging in normal and cancer cells with quantum dot immunoconjugates. Cell Res., 2003, 13(6): 503-507.

[51] A.F. van Driel, G. Allan, C. Delerue, et al., FrequencyDependent Spontaneous Emission Rate from CdSe and CdTe Nanocrystals: Influence of Dark States. Phys. Rev. Lett., 2005, 95(23): 236804.

[52] M. Mandal, S. Kundu, S.K. Ghosh, et al., Magnetite 
nanoparticles with tunable gold or silver shell. J. Colloid Interface Sci., 2005, 286(1): 187-194.

[53] L.P. Ramirez, K. Landfester, Magnetic polystyrene nanoparticles with a high magnetite content obtained by miniemulsion processes. Macromol. Chem. Phys., 2003, 204(1): 22-31.

[54] A.H. Lu, E.L. Salabas, F. Schüth, Magnetic Nanoparticles: Synthesis, Protection, Functionalization, and Application. Angew. Chem. Int. Ed., 2007, 46(8): 1222-1244.

[55] R.A. Frimpong, J. Dou, M. Pechan, et al., Enhancing remote controlled heating characteristics in hydrophilic magnetite nanoparticles via facile co-precipitation. $J$. Magn. Magn. Mater., 2010, 322(3): 326-331.

[56] J. Duan, L. Song, J. Zhan, One-pot synthesis of highly luminescent CdTe quantum dots by microwave irradiation reduction and their $\mathrm{Hg} 2+$-sensitive properties. Nano Res., 2010, 2(1): 61-68.

[57] T. Lühmann, M. Rimann, A.G. Bittermann, et al., Cellular uptake and intracellular pathways of PLL-g-PEG-DNA nanoparticles. Bioconjug. Chem., 2008, 19(9): 1907-1916.

[58] R. Bhadelia, A. Bogdan, R. Kaplan, et al., Cerebrospinal fluid pulsation amplitude and its quantitative relationship to cerebral blood flow pulsations: a phase-contrast MR flow imaging study. Neuroradiology, 1997, 39(4): 258264.

[59] M. Freund, M. Adwan, H. Kooijman, et al., [Measurement of CSF flow in the spinal canal using MRI with an optimized MRI protocol: experimental and clinical studies]. ROFO. Fortschr. Geb. Rontgenstr. Nuklearmed., 2001, 173(4): 306-314.

[60] A.C. Lui, T.Z. Polis, N.J. Cicutti, Densities of cerebrospinal fluid and spinal anaesthetic solutions in surgical patients at body temperature. Can. J. Anaesth., 1998, 45(4): 297-303.

[61] J. Chatterjee, Y. Haik, C.J. Chen, Size dependent magnetic properties of iron oxide nanoparticles. J. Magn. Magn. Mater., 2003, 257(1): 113-118.

[62] H. Itoh, T. Sugimoto, Systematic control of size, shape, structure, and magnetic properties of uniform magnetite and maghemite particles. J. Colloid Interface Sci., 2003, 265(2): 283-295

[63] J.A. Dearing, P.M. Bird, R.J.L. Dann, et al., Secondary ferrimagnetic minerals in Welsh soils: a comparison of mineral magnetic detection methods and implications for mineral formation. Geophys. J. Int., 1997, 130(3): 727736 .

[64] V.I. Klimov, A.A. Mikhailovsky, S. Xu, et al., Optical Gain and Stimulated Emission in Nanocrystal Quantum
Dots. Science, 2000, 290(5490): 314-317.

[65] N.M. Park, C.J. Choi, T.Y. Seong, S.J. Park, Quantum Confinement in Amorphous Silicon Quantum Dots Embedded in Silicon Nitride. Phys. Rev. Lett., 2001, 86(7): 1355-1357.

[66] A. Misra, S. Ganesh, A. Shahiwala, et al., Drug delivery to the central nervous system: a review. $J$ Pharm Pharm Sci, 2003, 6(2): 252-273.

[67] R.G. Thorne, W.H.I. Frey, Delivery of Neurotrophic Factors to the Central Nervous System: Pharmacokinetic Considerations. Clin. Pharmacokinet., 2001, 40(12): $907-$ 946.

[68] E. Dulkeith, A.C. Morteani, T. Niedereichholz, et al., Fluorescence Quenching of Dye Molecules near Gold Nanoparticles: Radiative and Nonradiative Effects. Phys. Rev. Lett., 2002, 89(20): 203002.

[69] E. Dulkeith, M. Ringler, T.A. Klar, et al., Gold Nanoparticles Quench Fluorescence by Phase Induced Radiative Rate Suppression. Nano Lett., 2005, 5(4): 585 589.

[70] C.C. Berry, Possible exploitation of magnetic nanoparticle?cell interaction for biomedical applications. J. Mater. Chem., 2005, 15(5): 543.

[71] I. Brigger, C. Dubernet, P. Couvreur, Nanoparticles in cancer therapy and diagnosis. Adv. Drug Deliv. Rev., 2012, 64, Supplement: 24-36.

[72] H. He, X. Sun, X. Wang, H. Xu, Synthesis of highly luminescent and biocompatible $\mathrm{CdTe} / \mathrm{CdS} / \mathrm{ZnS}$ quantum dots using microwave irradiation: a comparative study of different ligands. Lumin. J. Biol. Chem. Lumin., 2014, 29(7): 837-845.

[73] H. Qian, C. Dong, J. Weng, J. Ren, Facile onepot synthesis of luminescent, water-soluble, and biocompatible glutathione-coated CdTe nanocrystals. Small Weinh. Bergstr. Ger., 2006, 2(6): 747-751.

[74] R.K. Singhal, M.E. Anderson, A Meister, Glutathione, a first line of defense against cadmium toxicity. FASEB $J$. Off. Publ. Fed. Am. Soc. Exp. Biol., 1987, 1(3): 220-223.

[75] H. Soo Choi, W. Liu, P. Misra, et al., Renal clearance of quantum dots. Nat. Biotechnol., 2007, 25(10): 1165-1170.

Copyright $(\mathcal{C} 2016$ Indu Venugopal, Sebastian Pernal, Taylor Fusinatto, David Ashkenaz and Andreas Linninger. This is an open-access article distributed under the terms of the Creative Commons Attribution License, which permits unrestricted use, distribution, and reproduction in any medium, provided the original author and source are credited. 